\title{
Muscular dystrophy meets protein biochemistry, the mother of invention
}

\author{
Steven D. Funk and Jeffrey H. Miner \\ Department of Medicine, Division of Nephrology, Washington University School of Medicine, St. Louis, Missouri, USA

\begin{abstract}
Muscular dystrophies result from a defect in the linkage between the muscle fiber cytoskeleton and the basement membrane (BM). Congenital muscular dystrophy type MDC1A is caused by mutations in laminin $\alpha 2$ that either reduce its expression or impair its ability to polymerize within the muscle fiber BM. Defects in this BM lead to muscle fiber damage from the force of contraction. In this issue of the $J C I$, McKee and colleagues use a laminin polymerization-competent, designer chimeric BM protein in vivo to restore function of a polymerization-defective laminin, leading to normalized muscle structure and strength in a mouse model of MDC1A. Delivery of such a protein to patients could ameliorate many aspects of their disease.
\end{abstract}

\section{Many pathways to muscular} dystrophy

The mechanism whereby a contractile multinucleated skeletal muscle fiber is linked to the basement membrane (BM) that ensheaths it represents a paradigm for our understanding of BM assembly and interactions between the cell and extracellular matrix in the context of human pathobiology. Multiple matrix proteins, cell-surface receptors, transmembrane accessory proteins, cytoskeletal linker proteins, and glycosylating enzymes are required for the establishment and maintenance of the large, integrated complex that tightly links the myofiber cytoskeleton to the sarcolemma (plasma membrane) and the sarcolemma to the skeletal muscle BM (1). Genetic defects that alter the expression, structure, or function of these proteins cause a devastating class of diseases, the muscular dystrophies.

Muscular dystrophies are characterized by the progressive degeneration and weakness of skeletal muscle that includes muscle fiber necrosis, regeneration, and fibrosis (2). The severity and rate of progression of muscular dystrophy can vary dramatically, from congenital forms to the more common delayed-onset form known as Duchenne muscular dystrophy (3). From a cell biological perspective, muscular dystrophies with a genetic basis can be divided into types that correspond roughly to the site of the protein or protein complex that is affected by mutation. Mutations that affect the muscle BM cause congenital muscular dystrophy; those that affect the cytoskeletal protein dystrophin cause Duchenne and Becker muscular dystrophy; and those that affect transmembrane proteins called sarcoglycans cause limb-girdle muscular dystrophy (3). Interestingly, mutations that impact the enzymes responsible for glycosylation of dystroglycan cause the dystroglycanopathies, which manifest as muscular dystrophies with variable degrees of severity and timing of onset, as well as variable CNS and eye defects (4). Dystroglycan serves as the functional centerpiece of the dystrophin-glycoprotein complex that links the cytoskeleton to the $\mathrm{BM}$ and, therefore, plays a crucial role in maintaining skeletal muscle health (5).

\section{Laminin and the basement membrane}

Like all BMs, the skeletal muscle fiber BM contains specific isoforms from four major matrix protein families: laminins, type IV

Related Article: p. 1075

Conflict of interest: J.H. Miner has received research support from Third Rock Ventures and Hoffmann-La Roche.

Reference information: / Clin Invest. 2017;127(3):798-800. https://doi.org/10.1172/JCI92847.

collagens, nidogens, and heparan sulfate proteoglycans. Laminins are large heterotrimeric glycoproteins composed of one $\alpha$, one $\beta$, and one $\gamma$ chain (6). The major laminin isoform of skeletal muscle $\mathrm{BM}$ is laminin $\alpha 2 \beta 1 \gamma 1$ (Lm211; Figure 1, left), while a structurally and functionally different isoform, laminin $\alpha 5 \beta 2 \gamma 1$, is found at the neuromuscular junction (7). Lm211 is a cruciform macromolecule that can selfpolymerize in vitro to form a network via the formation of intermolecular ternary complexes involving the laminin N-terminal (LN) domain of each of the three chains (8). The unique $\mathrm{C}$-terminus of the $\alpha 2$ chain, called the laminin globular (LG) domain, binds to receptors on the surface of the myofiber, including $\alpha$-dystroglycan and integrin $\alpha 7 \beta 1$ (1). The binding of laminin to its receptors as muscle forms is thought to have a dual purpose: a) initiation of BM formation by concentrating the laminin at the cell surface above a critical concentration that promotes polymerization; and $b$ ) sending a signal to the cell (9). Upon maturation, laminin binding to its receptors links the muscle fiber to the BM, into which the laminin has become deeply integrated via direct and indirect interactions with nidogen, collagen IV, and proteoglycans.

Mutations in the laminin $\alpha 2$-encoding gene (LAMA2) cause congenital muscular dystrophy. Mutations that prevent production of the protein typically (though not always) cause more severe disease than do missense mutations, internal deletions, or mutations that reduce Lm211 protein levels (1) (Figure 1, middle). The existence of several naturally occurring and engineered mouse models with Lama2 mutations with features of human congenital muscular dystrophy have been instrumental for investigating pathogenic mechanisms and potential treatments for both severe and milder forms of the disease (10). issue (11) and previous studies have exploit- 


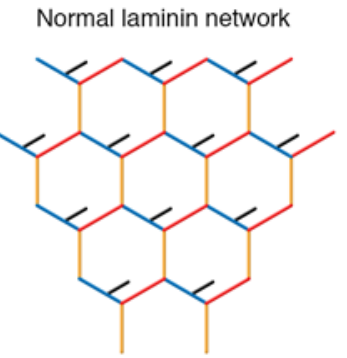

$d y^{2} / d y^{2 s}$ laminin network

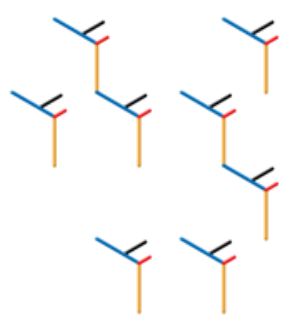

$\psi$ Truncated $\alpha$
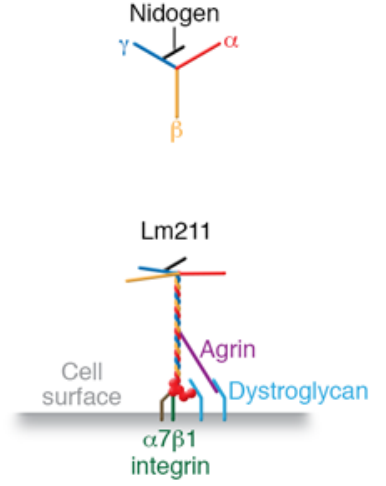

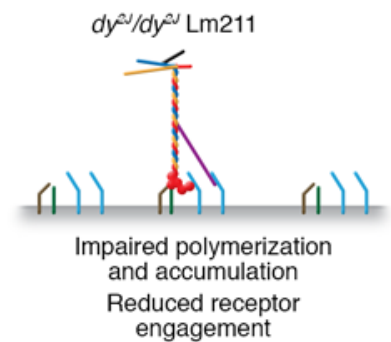

engagement
Repaired laminin network

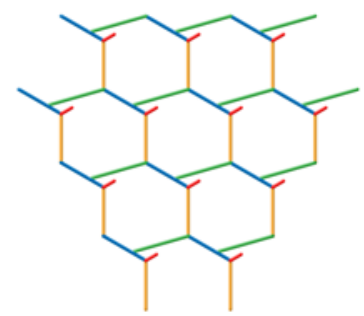

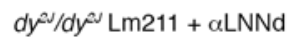

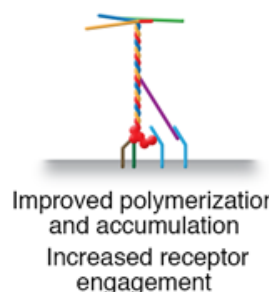

Figure 1. A designer chimeric polypeptide restores function of defective laminin. Lm211 is a heterotrimeric glycoprotein consisting of one $\alpha 2-$, one $\beta 1$-, and one $\gamma 1$-subunit that is an essential extracelIular matrix component of the skeletal muscle fiber BM. WT Lm211 readily polymerizes and becomes integrated into the $\mathrm{BM}$ and serves to link the muscle fiber to the $\mathrm{BM}$ via receptor binding. Mutations in the gene encoding the laminin $\alpha 2$-subunit cause a congenital muscular dystrophy due to absent or defective Lm211. The mutant Lm211 present in $d y^{2 /} / d y^{2 /}$ mice has a truncated laminin $\alpha 2$ that is unable to polymerize. The polymerization defect results in disruption of the BM, leading to loss of BM integrity and reduced muscle function. In this issue, McKee and colleagues developed a chimeric polypeptide ( $\alpha$ LNNd) that contains the laminin N-terminal (LN) domain of the laminin $\alpha 1$-subunit and the G2-G3 domains of nidogen, which simultaneously rescued polymerization of mutant truncated laminin $\alpha 2$ / Lm211 (right) and linked laminin to the collagen IV network (not depicted). $\alpha$ LNNd thereby restored muscle function in $d y^{2 /} / d y^{2 /}$ mice.

ed the intermolecular interactions among $\mathrm{BM}$ proteins and their cognate receptors that have been deciphered through decades of basic science research. In addition to laminin-receptor interactions, laminins are secondarily and indirectly linked to cellsurface receptors by the heparan sulfate proteoglycan agrin, which binds to the $\mathrm{LM} \gamma 1$ coiled-coil region via its $\mathrm{N}$-terminal agrin domain as well as to dystroglycan and integrins via its C-terminal LG-like domains (12). Furthermore, the laminin network is linked to the type IV collagen network by nidogen, which binds to the short arm LE3b segment of $L M \gamma 1$ via nidogen's $G 3$ domain and to a region in collagen IV near the Cterminal noncollagenous 1 (NC1) domain via nidogen's G2 domain (13).

Previously, research teamsled by Ruegg tested a molecular therapy in severe forms of congenital muscular dystrophy, in which
$\mathrm{LM} \alpha 2$ is either truncated and expressed at very low levels $\left(d y^{w /} / d y^{w}\right.$ mice) or completely missing $\left(d y^{3 K /} d y^{3 K}\right.$ mice). These mice and patients who lack LM $\alpha 2$ exhibit increased LM $\alpha 4-$ and LM $\alpha 5$-containing trimers, but decreased $\alpha$-dystroglycan expression. Although these laminins have poor musclespecific receptor binding, and LM $\alpha 4$ lacks the LN domain required for polymerization, Ruegg and colleagues hypothesized that a mini-agrin protein containing only the laminin- and dystroglycan-binding domains might both stabilize the laminin network and restore dystroglycan levels. Collectively, $d y^{3 K /} d y^{3 K}$ and $d y^{\mathrm{w}} / d y^{\mathrm{w}}$ mice expressing mini-agrin in skeletal muscle via a transgene exhibited a marked increase in muscle function and lifespan and improved BM morphology, with increased incorporation of the polymerization-capable LM $\alpha 5$. In this scenario, mini-agrin links the lami- nin network, albeit an aberrant network, to dystroglycan, which is likely the major mechanism of disease attenuation $(14,15)$.

Patients who harbor mutations that only affect the LM $\alpha 2$ LN domain have mild phenotypes compared with those with mutations that result in total loss of LM $\alpha 2$ (16), similar to missense mutations in the LM $\beta 2$ LN domain that affect the kidney glomerular BM of Pierson syndrome spectrum patients (17). On the basis of biochemical studies, LN domain mutations in LM $\beta 2$ and LM $\alpha 2$ are predicted to confer some degree of laminin polymerization defects. For LM $\alpha 2$, these are collectively modeled by $d y^{2 /} / d y^{2 J}$ mice that lack the LM $\alpha 2$ LN domain; but, unlike $d y^{w} / d y^{w}$ mice, $d y^{2 /} / d y^{2 J}$ mice show only a modest decrease in LM $\alpha 2$ protein levels. McKee et al. exploited the laminin-nidogen-collagen linkage to explore the therapeutic efficacy of a chimeric fusion protein, $\alpha \mathrm{LNNd}$, via transgenic expression in skeletal muscle of $d y^{2 /} / d y^{2 J}$ mice.

The aLNNd protein is composed of the LM $\alpha 1$ LN and LEa1-4 domains fused to the G2-G3 portions of nidogen, thereby replacing the nidogen $\mathrm{G} 1$ domain and short segment that connects G1 to the G2-G3 segment with the LM $\alpha 1$ N-terminus. The nidogen portion binds to LM $\gamma 1$ LE3b via the G3 domain and to the collagen type IV network via the G2 domain, thereby preserving the laminin-collagen linkage. Biochemical studies and naturally existing laminin heterotrimer combinations (Lm111, Lm211) prove that both $\mathrm{LM} \alpha 1$ and $\mathrm{LM} \alpha 2$ LN domains facilitate polymerization through interactions with LM $\beta 1$ and LM $\gamma 1$ LN domains. Additionally, both overexpression and exogenous provision of Lm111 can ameliorate muscular dystrophy in Lama2-mutant mice (18, 19). Thus, the LM $\alpha 1 \mathrm{LN}$ domain of $\alpha \mathrm{LNNd}$ readily substitutes for the missing $\operatorname{LM} \alpha 2$ LN domain both in vivo and in vitro (11). This chimeric protein corrects the polymerization defect in $d y^{2 /} / d y^{2 J}$ mice (Figure 1 , right) (and should presumably do the same in LM $\alpha 2$ polymerization-defective patients), thereby reinforcing the concept that laminin polymerization is critical for maintaining BM integrity. Moreover, like the effects of the mini-agrin protein in the $d y^{w} / d y^{w}$ and $d y^{3 K} / d y^{3 K}$ models, the $\alpha$ LNNd chimera restored muscle performance in $d y^{2 J} / d y^{2 J}$ animals to a level similar to that 
of control animals and normalized most histopathological parameters. $\alpha \mathrm{LNNd}$ also normalized mutant $\mathrm{LM} \alpha 2$ and $\mathrm{LM} \gamma 1$ protein levels in vivo to those of control mice and restored polymerization of the mutant Lm211 on the surface of cultured cells, indicating that the fusion protein behaved as predicted.

\section{Prospects for the future}

There are currently no effective treatments for muscular dystrophies. Experimental studies reveal that pharmaceutical modulation of some basic physiological and cell biological processes, including inflammation, fibrosis, apoptosis, and regeneration, may improve patients' phenotypes (1). In parallel, we summarize here exciting, basic science-driven molecular modulations that address the matrix protein deficiencies underlying congenital muscular dystrophies. Although McKee et al. did not investigate inducible rescue with $\alpha \mathrm{LN}$ $\mathrm{Nd}$, which would better mimic attempted therapy in a clinical context, the efficacy of induced expression of mini-agrin (20) indicates that $d y^{2 J} / d y^{2 J}$ phenotypes would likely be improved by late therapeutic intervention. In addition, Qiao et al. demonstrated that expression of a mini-agrin cDNA packaged into an adeno-associated virus that can either be directly injected into muscles or delivered systemically improves phenotypes in mice (21). Interestingly, $\alpha \mathrm{LNNd}$ did not interfere with WT Lm111 polymerization in assays on myotubes in vitro (11), suggesting that this fusion protein does not perturb normal BM integrity in vivo. We would further suggest that the combination of mini-agrin and $\alpha \mathrm{LNNd}$ may have synergistic effects by enhancing dystroglycan binding of an $\alpha \mathrm{LNNd}$-stabilized LM $\alpha 4$ network, the most highly compensating laminin in patients and mice lacking $\mathrm{LM} \alpha 2$. The study by Mckee et al. indicates that functional designer molecules, potentially in combination with suit- able delivery systems and pharmaceutical strategies (1), hold promise as future therapeutics for congenital muscular dystrophy. Importantly, the small sizes of $\alpha$ LNNd (157 $\mathrm{kDa})$ and mini-agrin (106 $\mathrm{kDa}$ ) relative to the affected $341-\mathrm{kDa} \mathrm{LM} \alpha 2$ chain and the approximately $750-\mathrm{kDa} \mathrm{Lm} 211$ trimer, together with the fact that $\alpha \mathrm{LNNd}$ and mini-agrin function as monomers, make them well suited for therapeutic intervention, whether delivered as naked or virally encoded proteins.

\section{Acknowledgments}

The authors were supported by NIH grants R01DK078314 and T32DK007126.

Address correspondence to: Jeffrey $\mathrm{H}$. Miner, Division of Nephrology, Washington University School of Medicine, 4523 Clayton Ave., St. Louis, Missouri 63110, USA. Phone 314.362.8235; E-mail: minerj@wustl.edu.

\footnotetext{
1. Durbeej M. Laminin- $\alpha 2$ chain-deficient congenital muscular dystrophy: pathophysiology and development of treatment. Curr Top Membr. 2015;76:31-60.

2. Rahimov F, Kunkel LM. The cell biology of disease: cellular and molecular mechanisms underlying muscular dystrophy. J Cell Biol. 2013;201(4):499-510.

3. Guiraud S, Aartsma-Rus A, Vieira NM, Davies KE, van Ommen GJ, Kunkel LM. The pathogenesis and therapy of muscular dystrophies. Annu Rev Genomics Hum Genet. 2015;16:281-308.

4. Godfrey C, Foley AR, Clement E, Muntoni F. Dystroglycanopathies: coming into focus. Curr Opin Genet Dev. 2011;21(3):278-285.

5. Michele DE, Campbell KP. Dystrophin-glycoprotein complex: post-translational processing and dystroglycan function. J Biol Chem. 2003;278(18):15457-15460.

6. Miner JH. Laminins and their roles in mammals. Microsc Res Tech. 2008;71(5):349-356.

7. Patton BL, Miner JH, Chiu AY, Sanes JR. Distribution and function of laminins in the neuromuscular system of developing, adult, and mutant mice. J Cell Biol. 1997;139(6):1507-1521.

8. Yurchenco PD, Cheng YS. Self-assembly and calcium-binding sites in laminin. A three-arm interaction model. J Biol Chem. 1993;268(23):17286-17299.
}

9. Colognato H, Winkelmann DA, Yurchenco PD. Laminin polymerization induces a receptorcytoskeleton network. J Cell Biol. 1999;145(3):619-631.

10. Saunier M, Bönnemann CG, Durbeej M, Allamand V, CMD Animal Model Consortium. 212th ENMC International Workshop: Animal models of congenital muscular dystrophies, Naarden, The Netherlands, 29-31 May 2015. Neuromuscul Disord. 2016;26(3):252-259.

11. McKee KK, Crosson SC, Meinen S, Reinhard JR, Rüegg MA, Yurchenco PD. Chimeric protein repair of laminin polymerization ameliorates muscular dystrophy phenotype. JClin Invest. 2017;127(3):1075-1089.

12. Meinen S, Ruegg MA. Congenital muscular dystrophy: mini-agrin delivers in mice. Gene Ther. 2006;13(11):869-870.

13. Ho MS, Böse K, Mokkapati S, Nischt R, Smyth N. Nidogens-Extracellular matrix linker molecules. Microsc Res Tech. 2008;71(5):387-395.

14. Bentzinger CF, Barzaghi P, Lin S, Ruegg MA. Overexpression of mini-agrin in skeletal muscle increases muscle integrity and regenerative capacity in laminin- $\alpha 2$-deficient mice. FASEB J. 2005;19(8):934-942.

15. Moll J, et al. An agrin minigene rescues dystrophic symptoms in a mouse model for congenital muscular dystrophy. Nature. 2001;413(6853):302-307.

16. Yurchenco PD. Integrating activities of laminins that drive basement membrane assembly and function. Curr Top Membr. 2015;76:1-30.

17. Matejas V, et al. Mutations in the human laminin $\beta 2$ (LAMB2) gene and the associated phenotypic spectrum. Hum Mutat. 2010;31(9):992-1002.

18. Rooney JE, Knapp JR, Hodges BL, Wuebbles $\mathrm{RD}$, Burkin DJ. Laminin-111 protein therapy reduces muscle pathology and improves viability of a mouse model of merosin-deficient congenital muscular dystrophy. Am J Pathol. 2012;180(4):1593-1602.

19. Gawlik K, Miyagoe-Suzuki Y, Ekblom P, Takeda S, Durbeej M. Laminin $\alpha 1$ chain reduces muscular dystrophy in laminin $\alpha 2$ chain deficient mice. Hum Mol Genet. 2004;13(16):1775-1784.

20. Meinen S, Barzaghi P, Lin S, Lochmüller H, Ruegg MA. Linker molecules between laminins and dystroglycan ameliorate laminin-alpha2deficient muscular dystrophy at all disease stages. J Cell Biol. 2007;176(7):979-993.

21. Qiao C, et al. Amelioration of laminin- $\alpha 2-$ deficient congenital muscular dystrophy by somatic gene transfer of miniagrin. Proc Natl Acad Sci U S A. 2005;102(34):11999-12004. 